\title{
Fiber Bragg grating sensors for clinical measurement of the first metatarsophalangeal joint quasi-stiffness
}

\author{
Pui Wah Kong, Chi Chiu Chan, Senior Member, IEEE, Marabelle Li-wen Heng, Yimin Liu, Yixuan \\ Leow, Daniel Tik-Pui Fong
}

\begin{abstract}
Assessing the mobility of the first metatarsophalangeal joint (MTPJ) of a human foot is useful in clinical practice but there are no standard methods of measurement. The present study developed a new instrumentation using Fiber Bragg grating (FBG) sensor and load cell to quantify the first MTPJ quasi-stiffness in a clinical setting. This system is portable, lightweight, and allows quantification of quasi-stiffness over different ranges of motion in both loading and unloading directions. The laboratory setting validation results showed that FBG sensors could measure MTPJ angular displacement with reasonably good accuracy. The proposed system was successfully trialed in a hospital setting operated by a clinician on eight human subjects. Non-linear torque-angular displacement relationship was observed in both loading and unloading phases, with varying MTPJ quasi-stiffness in the early [loading 6.30 (2.62) $\mathrm{Nmm} /{ }^{\circ}$ unloading 8.46 (2.29) $\mathrm{Nmm} /{ }^{\circ}$ ], middle [loading $7.13(2.17) \mathrm{Nmm} /{ }^{\circ}$, unloading $11.11(2.94) \mathrm{Nmm} /{ }^{\circ}$ ], and late [loading $24.54(7.14) \mathrm{Nmm} /{ }^{0}$, unloading $14.50(4.77) \mathrm{Nmm} /{ }^{\circ}$ ] ranges of motion. The new method for measuring the first MTPJ quasi-stiffness established in the present study serves as a reference and opens up opportunities for future clinical investigations.
\end{abstract}

Index Terms - optical fiber, foot, first toe, metatarsal, phalange, range of motion.

The study was funded by the Singapore Ministry of Education Academic Research Fund, Tier 1 Grant. We also wish to acknowledge the support from Nanyang Technological University under the Undergraduate Research Experience on CAmpus (URECA) programme. The funding sources did not involve in the conduct of the research or preparation of the article.

P. W. Kong is with the Physical Education and Sports Science Academic Group, National Institute of Education, Nanyang Technological University. Singapore (email: puiwah.kong@nie.edu.sg).

C. C. Chan is with the Sino-German College of Intelligent Manufacturing, Shenzhen Technology University, China (email: ccchan@sztu.edu.cn).

M. L. Heng is with the Podiatry Department, Singapore General Hospital, Singapore and the School of Health Sciences, University of South Australia, Australia (email: marabelle.heng.l.w@sgh.com.sg).

Y. Liu was with the School of Chemical and Biomedical Engineering, Nanyang Technological University, Singapore (email: liuyiminjl@163.com).

Y. Leow is with the School of Mechanical and Aerospace Engineering, Nanyang Technological University, Singapore (email: YLEOW005@e.ntu.edu.sg).

D. T. P. Fong is with the National Centre for Sport and Exercise Medicine, School of Sport, Exercise and Health Sciences, Loughborough University, United Kingdom (email: D.T.Fong@lboro.ac.uk).

\section{INTRODUCTION}

$\mathrm{T}$ HE human foot typically comprises 26 bones and 33 joints supported by many muscles, tendons and ligaments. In clinical practice, measuring the mobility of the first metatarsophalangeal joint (MTPJ) is useful for assessing hallux valgus surgery outcomes [1], screening of diabetic foot ulceration risks [2], and monitoring plantar fasciitis conditions [3]. However, standard methods for measuring first MTPJ mobility do not exist at present. Common practices adopted by clinicians include subjectively "feeling" how easily the MTPJ moves though its range of motion, visually estimating or measuring MTJP range of motion using goniometric devices $[1,3,4]$. Unfortunately, these traditional methods are not sufficiently reliable regardless of the experience of the testers [5] and clinicians have yet to come to a consensus on how foot joint mobility should be assessed.

To overcome the problems with subjectivity associated with clinical examination, new instrumentation to quantify MTPJ stiffness have been proposed in recent years. Rao [6] constructed a jig to measure the applied torque and resulting first MTPJ angular excursion to reflect the flexibility of the joint with the subjects in a standing position. Similarly, Man [7] developed a mechanical device to measure the MTPJ stiffness in sitting and standing positions using a dynamometer to control the speed over $40^{\circ}$ range of toe dorsi-flexion. Since these earlier devices are bulky and impractical for clinical practice, Farhadi [8] proposed an enhanced design of a light-weight, portable measuring unit that can be strapped onto the subject's foot to measure MTPJ stiffness. One method that most closely represents the clinical examination practice is the approach by Heng [9] who used video analysis together and a sleeve embedded with pressure sensor on the clinician's thumb. This method does not require any device attached to the subject and allows the quantification of MTPJ quasi-stiffness in a non-weight-bearing position on an examination table as in a regular clinical examination routine. There is, however, room for improvement in the accuracy of the force measurement and synchronisation of force and angular displacement data. 
The four methods described above used torque and angular displacement measurements to calculate the linear slope of the graph (torque-angle, or angle-torque) to indicate joint quasi-stiffness or flexibility. It is interesting to note that each of the four studies used a different method to calculate the slope of the graph. Fahadi [8] used regression analysis to find the best fit line for all torque-angle data points. Man [7] divided the maximum toque by the full range of $40^{\circ}$ to represent passive MTPJ stiffness. Rao [6] calculated the slopes only during the early (first 25\%) and late (last 25\%) phases but not the middle portion. In contrast, Heng [9] excluded data near the end points and used only the middle 'working range-of-motion' to calculate the slope during the unloading phase. These inconsistencies among studies suggested the MTPJ quasi-stiffness over different ranges of motion may have different clinical implications, since the non-linear and varying stiffness in human joints had been long recognized [10,11]. In the ankle joint, for example, it is useful to quantify the stiffness over the maximum, normal, and functional range of motion in both plantarflexion and dorsiflexion directions for screening of diabetic risks [11]. To our best knowledge, there is currently no clinical system that can measure MTPJ quasi-stiffness over different ranges of motion in both loading and unloading directions.

The purpose of this study was to develop new instrumentation that is practical for clinical use and can accurately measure the first MTPJ quasi-stiffness over different ranges in loading and unloading phases. The system should be portable, lightweight, and simple such that clinicians can operate the procedures easily.

\section{METHODS}

\section{A. Development of The System}

To measure quasi-stiffness, MTPJ angular displacement and the torque applied need to be quantified. We propose a system to simultaneously measure MTPJ range of motion using fiber Bragg grating (FBG) technology and the force applied by a clinician using a load cell (Sensorcraft Technology (S), Pte Ltd, Singapore, Fig. 1). The load cell weights less than $1.50 \mathrm{~kg}$ and has a dimension of $51 \mathrm{~mm} \times 17 \mathrm{~mm} \times 19 \mathrm{~mm}$. It can operate under a wide temperate range from -55 to $90^{\circ} \mathrm{C}$, with $110 \mathrm{~N}$ maximum load capacity for tension and compression. The nonlinearity / hysteresis fall with $+/ 0.05 \%$. The center wavelength of FBG ranges from $1540 \mathrm{~nm}$ to $1560 \mathrm{~nm}$, typically as $1550 \mathrm{~nm}$. The reflectivity of FBG was more than $90 \%$. The length of the grating was $3 \mathrm{~cm}$ coated with acrylate. The cable embedded with FBG nerves is unaffected by random wrinkling at the joints and allows unconstrained motion monitoring of joint displacement.

FBG is a type of distributed Bragg reflector constructed in a short segment of optical fiber that reflects particular wavelengths of light and transmits all others. This is achieved by creating a periodic variation in the refractive index of the fiber core [12]. FBG can therefore be used as an inline optical reflector to reflect specific wavelengths (central wavelength or resonant wavelength). We can track the drift of the FBG central (a)

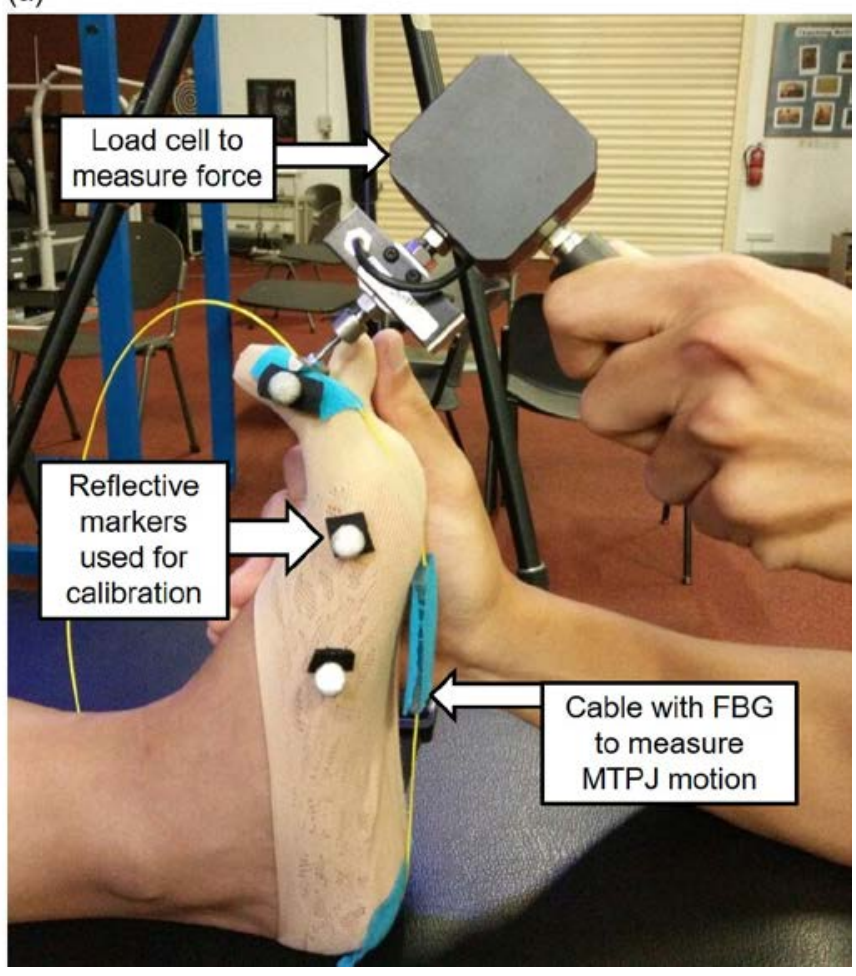

(b)

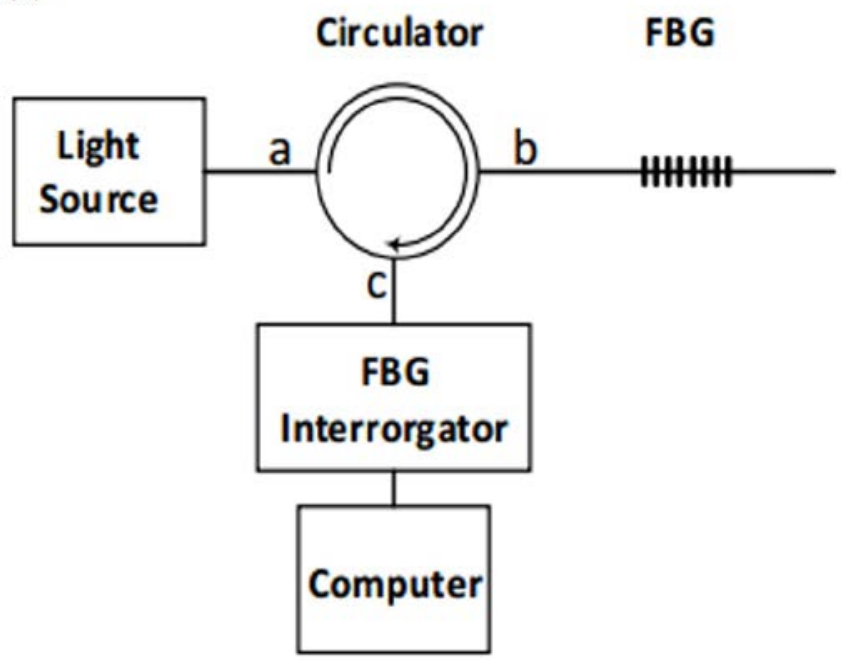

Fig. 1. (a) Fiber Bragg grating (FBG) device attached to the foot to sense the first metatarsophalangeal (MTPJ) motion, with a load cell to measure the force applied by a clinician. (b) Schematic representation of FBG system setup.

wavelength to measure the changes of physical parameters such as temperature and strain.

The use of FBG has been proven to be an excellent tool for measuring bending and strain [13] due to its unique properties such as being lightweight, flexible, and immune to electromagnetic interference. The central wavelength of the reflected wavelength must satisfy the Bragg condition:

$\lambda_{\text {ref }}=2 n_{\text {eff }} \Lambda$

where $n_{\text {eff }}$ is the core refractive index and $\Lambda$ is the period of the 
index of refraction variation of FBG. Because parameters $n_{\text {eff }}$ and $\Lambda$ exhibit a strong strain dependency, any changes of the strain in the FBG sensor region result in changing of $n_{\text {eff }}$ and $\Lambda$. The measurement of the strain can be determined by the drift of the Bragg wavelength $\lambda_{\text {ref. }}$ The FBG sensor region (producing an initial wavelength measurement $\lambda_{1}$ ) is coated with a polydimethylsiloxane (PDMS) membrane [14] to protect the grating from excess strain damage (Fig. 1(a)). The working principle of the device is illustrated in Figure 1b. The light source (Dense light, DL-BP1-1501A, with the wavelength range from 1200-1700 nm) emits light waves which enter the circulator (3 ports with SMF-28 fiber pigtails) at (a) and exit at (b) towards the FBG sensor region. The Bragg grating reflects a stable and specific wavelength of light that re-enters the circulator at (b) and exits at (c) towards the interrogator (Ibsen, I-MOM 512 USB, with a spectral resolution $0.5 \mathrm{pm}$ ), which deciphers the light wave and displays its wavelength and intensity on the screen. When the strain is applied to FBG, both the period of FBG and the refractive index of fiber core will change. As the strain increases, the FBG reflection peak drifts in the direction of the long wave.

To apply the system on human subjects, the subject first worn a disposable sock for hygiene reason. With the foot resting in a neutral position, the FBG sensor is attached on the disposable sock garment at the first toe and the heel regions of the foot using Velcro strips to ensure tautness (Fig. 1(a)). Specifically, the sensor is anchored on the plantar first proximal phalanx and plantar heel near the Achilles tendon. The clinician holds the foot stable with one hand and uses the load cell to apply a dorsi-flexion force normal to the first toe, inducing MTPJ motion. The FBG sensor experiences some strain as the angular displacement of the MTPJ increases, and this results in a shift in the wavelength of light reflected by the grating. A custom software was developed to acquire data from the load cell and FBG system simultaneously (Fig. 3).

\section{B. Calibration and Validation}

The calibration experiment was approved by the Nanyang Technological University Institutional Review Board (IRB-2016-11-032). To obtain calibration equations for the FBG sensors, 10 female subjects with no known foot conditions were recruited. The inclusion criteria of the subjects were: 1) aged 21 to 45 years, 2) free from any foot injury at the time of the study, 3) no injuries in the foot that resulted in a rest period of 7 days or more during the last 6 months, 4 ) no history of foot surgery, and 5) not have any rheumatological or connective tissue conditions. Subjects were excluded if they had gone through any surgeries to the foot, had serious foot injuries, experienced discomfort or pain of the foot at the time of the study, or self-reported any foot deformities (e.g. bunions). All subjects provided written informed consent to participate in the study.

In a laboratory setting, each eligible subject wore a new pair of disposable garment and laid in a supine position on an examination table [9]. The cable embedded with FBG sensors was attached to the testing foot. Three retro-reflective markers were adhered to the medial boarder of the foot in order to allow motion tracking of the joint movements (Fig. 1(a)). A three-dimensional (3D) motion capture system with four

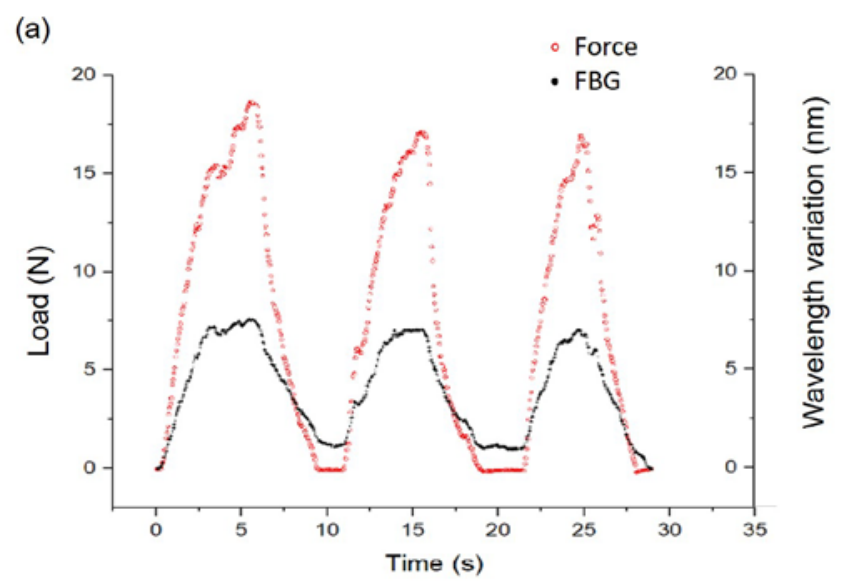

(b)

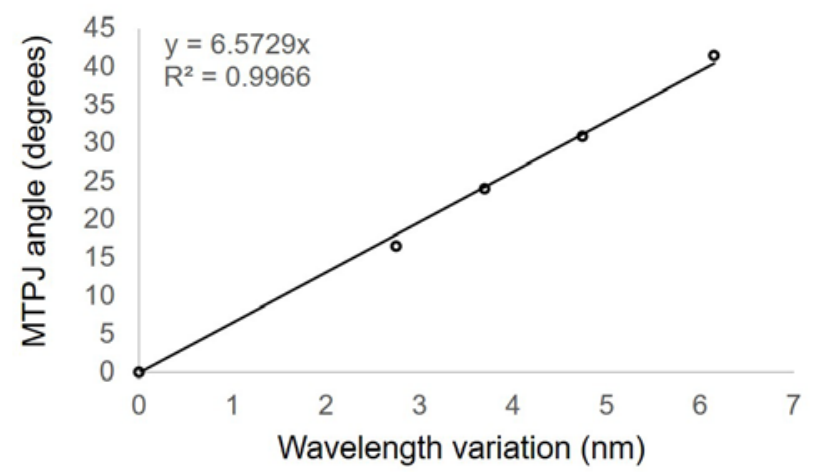

Fig. 2 (a). Synchronized force and fibre Bragg gratings (FBG) wavelength shift $(\mathrm{nm})$ recorded during three repetitions of loading and unloading the first metatarsophalangeal joint. (b) An example of linear relationship between metatarsophalangeal joint (MTPJ) angle and FBG wavelength variation (subject 9 , right foot).

infrared cameras (Vicon, Oxford, UK) was used as the gold standard to track the marker positions and to calculate the first MTJP angular displacements.

The following calibration procedures were performed on each subject. These procedures were used based on the study by Heng et al. [9] in which quasi-stiffness of subjects were measured using a tactile pressure sensing system. A tester applied a dorsiflexion force to flex the first toe at four intervals, gradually increased to maximum degree of dorsiflexion. At each interval, the first toe was kept stationary for several seconds so that stable wavelengths drift of the FBG could be recorded. This method of pausing the first toe at multiple intervals along the working range of motion closely followed that described by Heng et al. [9] for stable MTPJ angle measurements via offline video analysis. As the toe moved, MTPJ motion was concurrently recorded by the FBG device and the 3D motion capture system. Both left and right feet were tested, each with three trials collected. In each trial, there were four data points of FBG wavelength drift corresponding to the 
actual MTPJ angular displacements obtained from the gold standard of 3D motion analysis. By fitting a linear line to the data, the relationship between the wavelength drift and the MTPJ angular displacement can be determined. While the relationship was highly linear for each individual trial (Fig 2b), it is necessary to determine a relationship that can be generally applied to other subjects rather than limiting to one's own data. To achieve this goal, the dataset out of three trials that produced the best fit (highest $\mathrm{R}^{2}$ ) was selected per foot per subject. Taking the best dataset from all 10 subjects, a calibration equation was obtained ( 4 points $\times 10$ subjects $=40$ data points) for each foot:

$$
\begin{array}{ll}
\theta_{\text {left }}=9.036 \Delta \lambda & {\left[\mathrm{R}^{2}=0.906\right]} \\
\theta_{\text {right }}=9.156 \Delta \lambda & {\left[\mathrm{R}^{2}=0.842\right]}
\end{array}
$$

where $\theta$ is the MTPJ angular displacement (in degrees) and $\lambda$ is the FBG wavelength shift (in $\mathrm{nm}$ ). With these calibration equations, future MTPJ angular displacement can be measured directly from Bragg reflection peak drift read from the FBG demodulator without the need of any 3D motion capture system.

To verify the accuracy of the calibration equations, independent datasets of maximum MTPJ displacement were obtained from all 10 subjects so that the values calculated from the FBG calibration equations can be checked against those measured from 3D motion analysis. In this validation experiment, a tester dorsiflexed the subject's first toe to the maximum extent and paused for a few seconds. MTJP motion was concurrently captured by the FBG system and the 3D motion analysis system. The wavelength drift data were substituted into the calibration equation to calculate maximum MTPJ angular displacement. To evaluate the accuracy, we calculated the root mean square (RMS) error of the MTPJ angular displacement calculated from the FBG calibration equations versus the gold standard of 3D motion analysis. For each subject, nine independent trials were taken per foot and the average RMS error value was used.

\section{Applications in a Clinical Setting}

After successful development and validation, the FBG system was applied for clinical use in a hospital setting whereby a podiatrist operated the system to measure the first MTJP quasi-stiffness of human subjects. The clinical study was approved by the Singhealth Centralised Institutional Review Board (CIRB Ref: 2017/2559). Eight female subjects provided written informed consent to participant in the clinical study [mean (SD), age $=29.4(7.6)$ yrs, mass $=59.9(9.7) \mathrm{kg}$, height $=$ $161.1(4.1) \mathrm{cm}]$. All subjects were free from foot injuries, previous foot surgery, and rheumatology or connective tissue conditions.

A trained podiatrist with 9 years of clinical experience conducted all the tests in a clinical examination room of the hospital. Each subject had their left foot measured using the FBG system operated by the podiatrist. The moment arm was measured (in $\mathrm{mm}$ ) from the tuberosity of the first metatarsal head to just beneath the tuberosity of the first distal phalange in the medial aspect of the foot [9]. The subject lied on an examination table and her tested foot was fitted with the FBG system. The podiatrist secured the testing foot in its neutral position by holding at the ankle using the non-working hand. Once the foot was stabilised, the podiatrist used the working hand to control the load cell to slowly dorsiflex the first toe to maximum range and then return to the neutral position (Fig. 3(a)). When handling the load cell, cation was taken to apply a normal force at the distal edge of the first phalanx to induce sagittal plane motion and prevent any unwanted abduction/addition movements. Previous work have indicated that the experience of the tester can influence the reliability of MTPJ quasi-stiffness measurements due to the difficulty in controlling the foot position and manipulating fine joint movements [9]. Thus, we engaged an experienced podiatrist across all clinical testings to ensure good consistency and fine manipulation of the foot joints.

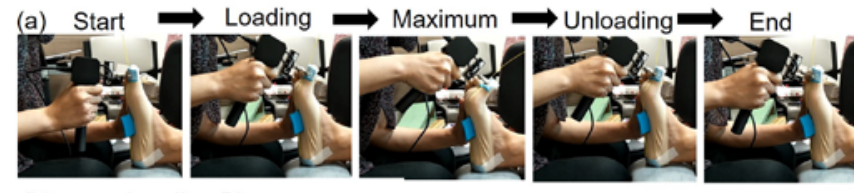

(b) Loading Phase
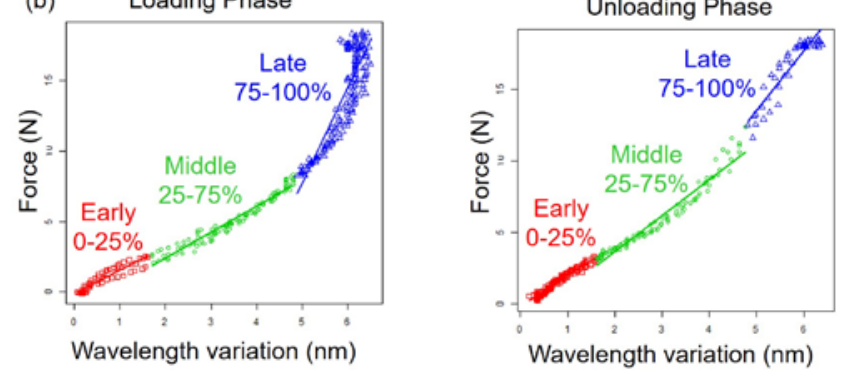

Fig. 3. (a) A podiatrist operating the load cell and FBG system to load and unload the first toe. (b) Due to the non-linear relationship, data were analysed over thee ranges for loading and unloading phases: Early 0-25\%, middle $25-75 \%$, and late $75-100 \%$.

The procedures were repeated three times on the foot. The FBG wavelength shift and the force applied by the podiatrist via the load cell were continuously recorded during both loading and unloading phases (Fig. 2(a)). With the torque (force $\times$ moment arm) and MTPJ angular displacement data, the quasi-stiffness (in $\mathrm{Nmm} /{ }^{\circ}$ ) can be calculated from the torque-angle graph (Fig. 3(b)). As expected in passive joint stiffness response, the torque versus angular displacement plots were non-linear [9,11]. Over the middle 'working range-of-motion', the quasi-stiffness was rather linear [9]. As the range of motion approaches the end range, the quasi-stiffness becomes stiffer (steeper gradient). There are also clear differences in the response between the loading and unloading phases, as previously reported in the ankle joint [11].

Given the nonlinear relationship between torque and MTPJ angular displacement, we calculated the slope at three different ranges (early $0-25 \%$, middle $25-75 \%$, late $75-100 \%$ ) from zero to maximum MTPJ displacement (Figure 3(b)). The slope of 
the loading and unloading phases were calculated separately, resulting in a total of six quasi-stiffness values per subject.

\section{Results}

In the validation experiment on 10 subjects, the mean (SD) RMS error of the FBG system were $12.4(8.9)^{\circ}$ for right foot and $14.6(8.0)^{\circ}$ for left foot, respectively, when compared with gold standard of 3D motion analysis (TABLE I).

TABLE I

ROOT-MEAN-SQUARED (RMS) ERROR OF THE FIRST METATARSOPHALANGEAL JOINT (MTPJ) MAXIMUM DORSIFLEXION CALCULATED USING FBG SYSTEM $(\mathrm{N}=10)$

\begin{tabular}{ccc}
\hline Subject & RMS error - right $\left({ }^{\circ}\right)$ & RMS error - left $\left({ }^{\circ}\right)$ \\
\hline S1 & 12.6 & 8.8 \\
S2 & 24.8 & 8.2 \\
S3 & 27.8 & 35.1 \\
S4 & 5.2 & 12.6 \\
S5 & 9.3 & 17.0 \\
S6 & 4.7 & 9.2 \\
S7 & 5.1 & 13.3 \\
S8 & 4.2 & 9.3 \\
S9 & 9.6 & 14.2 \\
S10 & 20.6 & 18.6 \\
Mean & 12.4 & 14.6 \\
SD & 8.9 & 8.0 \\
\hline \hline
\end{tabular}

S1 to S10 rep resent individual subjects in the validation experiment.

In the clinical study, a non-linear torque-angular displacement relationship was observed in all eight subjects. The MTPJ quasi-stiffness varied substantially over the early, middle, and late ranges and also differed between the loading and unloading directions (TABLE II). In general, the quasi-stiffness increased with the MTPJ range of motion, with the steepest slope occurred in the late (75-100\%) loading phase.

TABLE II

FIRST METATARSOPHALANGEAL JOINT (MTPJ) QUASI-STIFFNESS (IN NMM/º) OVER DIFFERENT RANGES IN UNLOADING AND UNLOADING PHASE $(\mathrm{N}=8)$

\begin{tabular}{cccc}
\hline \hline Phase & Early (0-25\%) & Middle (25-75\%) & Late (75-100\%) \\
\hline Loading & $6.30(2.62)$ & $7.13(2.17)$ & $24.65(7.14)$ \\
Unloading & $8.46(2.29)$ & $11.11(2.94)$ & $14.50(4.77)$ \\
\hline \hline
\end{tabular}

\section{DISCUSSION}

This study developed new clinical system for measuring the first MTPJ quasi-stiffness using FBG sensor coupled with a load cell. In the validation experiment, the accuracy of the FBG sensors for quantifying MTPJ angular displacement was reasonably good as compared with the gold standard of 3D motion analysis. The new system has been successfully trialed in a hospital setting, operated by a clinician to measure the first MTJP quasi-stiffness on human subjects. Using this system in a clinical setting, this study is the first to report MTPJ quasi-stiffness over different phases in both loading and unloading directions.

The FBG system developed in this study is practical for clinical use and can measure the first MTPJ quasi-stiffness over different ranges in the loading and unloading phases. Earlier laboratory devices for measuring MTPJ mobility tended to be heavy and large in size [6,7]. Compared with these bulky devices which are impractical for clinical use, both the load cell and FBG embedded cable in the present study are portable, small in size, and lightweight. While Farhadi et al. [8] also proposed a portable mechanical device which is small and light, the measuring unit to be strapped onto the subject's foot - the comfort of wearing this device is unknown. In addition, the fitting of the unit requires adjustment of load cell positions by lead screws and reconfiguration of left/right foot via rotation and spring loaded joint. These procedures may be challenging for clinicians to operate without technical support. The new system proposed in our study can overcome some of these limitations as the FBG embedded cable is soft and flexible, allowing unconstrained joint motion without causing discomfort. The cable can be attached easily onto the subject's sock garment by clinicians using Velcro or tapes without mechanical parts such as screw or spring. Our new approach offers a more comfortable for the patients and more user-friendly for the clinicians than earlier methods. With simple instructions, this device can be operated by healthcare aides such as assistants and technicians to lower the operational cost of the healthcare system.In the clinical study, the first MTPJ quasi-stiffness values measured on eight female subjects ranged from $6.30 \mathrm{Nmm} /{ }^{\circ}$ to $24.65 \mathrm{Nmm} /{ }^{\circ}$ (TABLE II). Over the middle 25 to $75 \%$ range, our quasi-stiffness values (loading $7.13 \mathrm{Nmm} /{ }^{\circ}$, unloading $11.11 \mathrm{Nmm} /{ }^{\circ}$ ) are comparable to the data (12.6 Nmm $/{ }^{0}$ to $\left.19.9 \mathrm{Nmm} /{ }^{\circ}\right)$ reported by Heng et al. [9] who measured MTPJ quasi-stiffness over the middle working-range-of-motion during the unloading phase in a non-weightbearing position. When compared with stiffness values measured under weightbearing or semi-weightbearing conditions, our data are lower than the average MTPJ passive stiffness of $29.42 \mathrm{Nmm} /{ }^{\circ}$ (sitting) and $80.28 \mathrm{Nmm} /{ }^{\circ}$ (standing) reported by Man et al [7]. We took measurements in a non-weightbearing position to approximate clinical examination routine, similar to the procedures used by Heng et al. [9]. Other studies used different calculation methods and units of measurement to indicate MTPJ flexibility (9.8 - 51.5 o/N.cm, [6]) or stiffness (3.49 $-14.42 \mathrm{Nmm} / \mathrm{kg}$ radian, [8]) and hence direct comparison of MTPJ quasi-stiffness values are not feasible.

In terms of the measurement accuracy, the average RMS error of the left and right sides was $13.5^{\circ}$. Based on a maximum MTPJ dorsiflexion range of $110^{\circ}$ previously reported in the literature [15], the error associated with the FBG system was $12.3 \%$. This magnitude of error is similar to those of MTPJ stiffness measured using a mechanical system, with average errors of $10.7 \%$ (left foot) and $10.8 \%$ (right foot) calculated from mean and standard error of measurement values [8]. In addition to validity, the reliability of a system is also of clinical importance [9]. The next logical step will be to conduct a test-retest reliability study to check if the measurements are repeatable on separate days. 
To our best knowledge, the present study is the first to measure MTPJ quasi-stiffness over different ranges of motion in both loading and unloading directions. This new analysis approach illustrated a clear non-linear relationship between torque and MTPJ angular displacement which cannot be revealed by simpler methods such as taking the maximum torque value [7] or fitting a line through data points in the entire range [8]. Calculating stiffness over selected range only is insufficient and may miss out important information. For example, Heng et al. [9] measured the first MTPJ quasi-stiffness during the middle working-range-of motion and ignored the data near the end points. Interesting, MTPJ flexibility near the end points (late 25\%) can successfully differentiate individuals with low, normal, and high foot arch [6]. For a comprehensive analysis, it is therefore better to calculate the slope over different ranges of the non-linear torque-angular displacement curve in both loading and unloading phases as performed in the present study. While we did not compare the first MTPJ quasi-stiffness among different groups of patients or healthy individuals, the new method established in the present study serves as a reference and opens up opportunities for future clinical investigations. The concepts and techniques developed in the present study for measuring MTPJ quasi-stiffness should be applicable to other joints such as finger and ankle joints.

There are a few limitations to the present study. First, the accuracy of FBG sensors to track MTPJ motion can be further improved. Currently, the FBG system output is sensitive to the tension of the cable attached to the disposable garment. Pulling the cable too tight will break the sensor or result in unresponsiveness. Technical improvement in standardizing the cable tension is essential for more accurate measurements of MTPJ angular displacement. Second, the FBG system was calibrated, validated, and applied on individuals with normal and healthy feet. Challenges may arise when using the system on individuals with foot deformities such as hallux valgus whereby the joint alignment deviates substantially from that of normal foot. Thirdly, one end of the FBG line is anchored to the plantar heel and the other end on the plantar first phalange. The varying width of forefoot amongst individual would result in angular differences between the anchoring points. Methods to account for such differences could increase accuracy of MTPJ quasi-stiffness measure.

\section{CONCLUSIONS}

This study reported a new instrumentation using FBG sensors and load cell to quantify the first MTPJ quasi-stiffness in a clinical setting. The validation results showed that FBG sensors can measure MTPJ motion with reasonably good accuracy. The proposed system was successfully implemented in a hospital setting operated by a clinician. Non-linear torque-angular displacement relationship was observed, with varying MTPJ quasi-stiffness in the early, middle and late range-of-motion in both loading and unloading directions. These objective quasi-stiffness measurements may guide clinicians in the evaluation of foot joint mobility.

\section{REFERENCES}

[1] B. Özkurt, C. N. Aktekin, M. Altay, O. Belhan, and Y. Tabak, "Range of motion of the first metatarsophalangeal joint after chevron procedure reinforced by a modified capsuloperiosteal flap," Foot \& Ankle International, vol. 29, no. 9, pp. 903-909, 2013.

[2] H. Pham, D. G. Armstrong, C. Harvey, L. B. Harkless, J. M. Giurini, and A. Veves, "Screening techniques to identify people at high risk for diabetic foot ulceration: a prospective multicenter trial," Diabetes Care, vol. 23, no. 5, pp. 606-611, 2000.

[3] R. H. Allen, and M. T. Gross, "Toe flexors strength and passive extension range of motion of the first metatarsophalangeal joint in individuals with plantar fasciitis," Journal of Orthopaedic \& Sports Physical Therapy, vol. 33, no. 8, pp. 468-478, 2003.

[4] M. M. Hopson, T. G. McPoil, and M. W. Cornwall, "Motion of the first metatarsophalangeal joint. Reliability and validity of four measurement techniques," Journal of the American Podiatric Medical Association, vol. 85, no. 4, pp. 198-204, 1995.

[5] A. M. Jones, and S. A. Curran, "Intrarater and interrater reliability of first metatarsophalangeal joint dorsiflexion: goniometry versus visual estimation," Journal of the American Podiatric Medical Association, vol. 102, no. 4, pp. 290-298, Jul. 2012

[6] S. Rao, J. Song, A. Kraszewski, S. Backus, S. J. Ellis, J. T. D. Md, and H. J. Hillstrom, "The effect of foot structure on 1st metatarsophalangeal joint flexibility and hallucal loading," Gait \& Posture, vol. 34, no. 1, pp. 131-137, May. 2011.

[7] H. S. Man, A. K. L. Leung, J. T. M. Cheung, and T. Sterzing, "Reliability of metatarsophalangeal and ankle joint torque measurements by an innovative device,” Gait \& Posture, vol. 48, pp. 189-193, 2016.

[8] F. Farhadi, M. Faraz, M. Heng, and S. Johnson, "An Ergonomic Testing System for the First Metatarsophalangeal Joint Stiffness," Journal of Biomechanical Engineering, vol. 140, no. 10, pp. 104501, 2018.

[9] M. L. Heng, Y. K. Chua, H. K. Pek, P. Krishnasamy, and P. W. Kong, “A novel method of measuring passive quasi-stiffness in the first metatarsophalangeal joint," Journal of Foot and Ankle Research, vol. 9, no. 1 , pp. 41, 2016.

[10] G. B. Salsich, M. J. Mueller, and S. A. Sahrmann, "Passive ankle stiffness in subjects with diabetes and peripheral neuropathy versus an age-matched comparison group," Physical Therapy, vol. 80, no. 4, pp. 352-362, 2000.

[11] S. G. Trevino, W. L. Buford Jr, T. Nakamura, A. J. Wright, and R. M. Patterson, "Use of a torque-range-of-motion device for objective differentiation of diabetic from normal feet in adults," Foot \& Ankle international, vol. 25, no. 8, pp. 561-567, 2004.

[12] K. O. Hill, and G. Meltz, "Fiber Bragg grating technology fundamentals and overview," Journal of Lightwave Technology, vol. 15, no.8, pp. 1263-1276, 1997.

[13] B. A. Tahir, J. Ali, and R. A. Rahman, "Strain measurements using fibre Bragg grating sensor," American Journal of Applied Science (Special Issue), pp. 40-48, 2005.

[14] L. Niu, L. H. Chen, Z. Q. Tou, C. S. Tay, Y. F. Lin, P. W. Kong, F. R. Ong, and C. C. Chan, "Kayaking paddle blade compression load distribution sensing system based on optical fiber with a polydimethylsiloxane membrane," Applied Optics, vol. 57, no. 6, pp. 1387-1392, 2018.

[15] M. M. Hopson, T. G. McPoil, and M. W. Cornwall, "Motion of the first metatarsophalangeal joint. Reliability and validity of four measurement techniques", Journal of the American Podiatric Medical Association, vol. 85, no.4, pp.198-204.

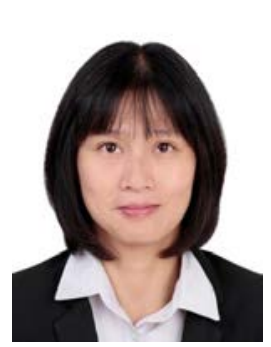

Kong Pui Wah (Veni) is an Associate Professor in the Physical Education and Sports Science Academic Group, National Institute of Education, Nanyang Technological University (NTU), Singapore. She obtained her Ph.D. in 2005 at Loughborough University, with a focus on computer simulation modelling in sports. She was an Assistant Professor at 
the University of Texas at El Paso (2005-2008) and a visiting faculty at the Emergency Responder Human Performance Lab, University of Pittsburgh School of Medicine (2008-2009), prior to joining NTU Singapore in 2009.

Her research expertise is biomechanics of human movement. She has published 60 peer-reviewed journal articles, primarily on sports and clinical biomechanics. Dr Kong has been appointed as an Associate Editor for Sports Biomechanics and Journal of Mechanics in Medicine and Biology. She also serves on the editorial board for Research in Sports Medicine. Working closely with industrial partners, hospitals, and schools, the work lead by Dr Kong's team at the Sports Biomechanics Laboratory have won national and international awards in the United Kingdom, United States, Taiwan, and Singapore.

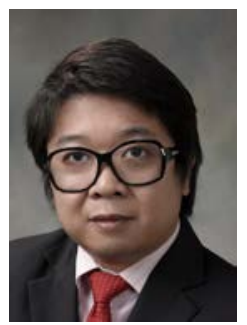

Chi Chiu Chan received the B.Eng. (Hons.) and Ph.D. degrees from Hong Kong Polytechnic University, Hong Kong, in 1996 and 2000, respectively. From 2000 to 2003, he was a Post-Doctoral Fellow with the Department of Electrical Engineering, Hong Kong Polytechnic University. He is currently an Associate Professor with the School of Chemical and Biomedical Engineering, Nanyang Technological University, Singapore. His research interests include optical fiber sensing system, fiber Bragg grating device, fiber optics chemical sensors, photonics crystal fiber biosensor, digital signal processing, such as artificial neural network, fuzzy logic, genetic algorithm, and wavelet, smart structures, and fiber optics chemical and biomedical sensing areas. His accomplishment in these areas is demonstrated in his 200 international publications.

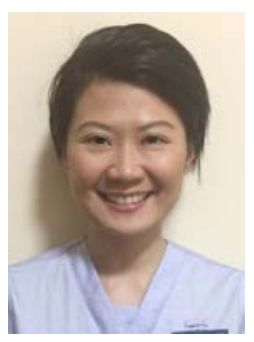

Marabelle Liwen Heng is principal podiatrist at Singapore General Hospital and adjunct lecturer, University of South Australia. Marabelle was conferred degree of Master of Science by research from Nanyang Technological University in 2015. The postgraduate study in foot biomechanics and joint hypermobility was completed under a full-time scholarship from Singapore Health Services Talent Development Fund. She is also a recipient of the Singapore General Hospital scholarship and graduated with a Bachelor of Podiatry degree from the University of South Australia in 2008.

Marabelle has been invited as faculty and speaker at various regional and international foot \& ankle conferences. Her clinical interests are in flatfoot and joint hypermobility. She was awarded the SingHealth Population-based, Unified, Learning System for Enhanced and Sustainable Health (PULSES) Centre Grant funded by the National Medical Research Council for a diabetes education study in 2018. Marabelle is involved in development of the profession through her involvement in Podiatry Association Singapore and the Chief Allied Health Officer's Office. Marabelle is currently serving her second term as president of Podiatry Association Singapore.

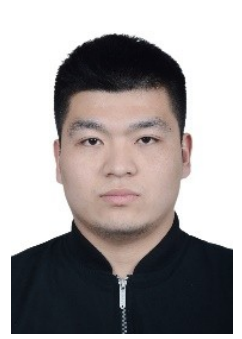

Yimin Liu was a Research Assistant (2017-2018) at the School of Chemical and Biomedical Engineering, Nanyang Technological University, Singapore. He obtained his Masters' Degree in optical Engineering from China Jiliang University in 2018. His main research interest is fiber Bragg Grating sensors.

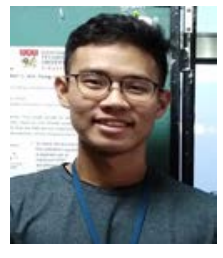

Yixuan Leow is an undergraduate student at the School of Mechanical and Aerospace Engineering, Nanyang Technological University, Singapore. He has an interest in mobility devices and is currently pursuing a Bachelors' Degree in Mechanical

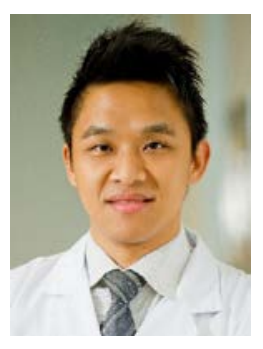
Engineering with design specialization.

Daniel T.P. Fong is a Senior Lecturer in Rehabilitation Science in the National Centre for Sport and Exercise Medicine, School of Sports, Exercise and Health Sciences, Loughborough University, UK. He obtained his $\mathrm{PhD}$ in 2008 at The Chinese University of Hong Kong, with a focus on orthopaedics sports medicine and biomechanics, and has been a Research Assistant Professor in the Department of Orthopaedics and Traumatology at The Chinese University of Hong Kong (2008-2013) and a Visiting Scholar at the Department of Orthopaedic Surgery at the University of Pittsburgh School of Medicine (2012), prior to joining Loughborough University in 2014.

His main research expertise is on the mechanism and prevention of sport-related ankle inversion sprain injury. He has published 60 peer-reviewed journal articles, primarily on sports medicine, orthopaedics and clinical biomechanics. He is currently the Editor-in-Chief of Sports Biomechanics Journal, and editorial board member in several other journals. 